\title{
An Approach of Exciting CNC Machine Tools Based on Worktable Empty Running Excitation
}

\author{
Mao Xinyong ${ }^{1, a}$, He Huanbin ${ }^{1, b}$, Liu Hongqi ${ }^{1, c}$, Li Bin ${ }^{1, d}$ and Qin Chao ${ }^{1, e}$ \\ ${ }^{1}$ School of Mechanical Science and Engineering, Huazhong University of Science and Technology, \\ Wuhan, Hubei, 430074, China \\ amaoxyhust@163.com, bhehuanbin@126.com, chustliuhq@163.com, \\ dlibin999@hust.edu.cn, e17771788410@163.com
}

\begin{abstract}
Keywords: machine tool vibration, modal parameters, worktable empty running excitation, feed speed, acceleration
\end{abstract}

Abstract. Dynamic characteristic of machine tool is an important factor which affects machining quality and machining efficiency. Traditional experimental modal analysis method requires the measured structure to be in a relatively static state. So the dynamic characteristics of machine tool structure cannot be measured in operating state. In this paper, an approach of exciting CNC machine tools based on worktable empty running excitation is proposed. The machine tool structure is excited by inertial force generated during the reciprocating motion of machine worktable. The motion of worktable is designed and the influencing factors of excitation are analyzed. The effect of feed speed and acceleration to excitation is analyzed through experiment. Compared to impact test in static state, the method is performed in running state. So it can reflect the dynamics of machine tools in operation accurately.

\section{Introduction}

Dynamic characteristic of the machine tool has become a very important factor affecting the performance of the machine. Traditional dynamics research method is experimental modal analysis (EMA) in a static state. In general, we strike the machine and measure the excitation force signal and response signal at the same time. Then we process the obtained signal and identify the modal parameters [1,2]. However, there's a great difference in dynamics of machine tool between operation state and static state. In operation state, the external conditions of the machine and the friction between various components change dynamically. Machine dynamics is also in the state of change $[3,4]$.

In order to study the dynamic characteristics of the machine in operation state, operational modal analysis (OMA) was introduced into the field of machine dynamics [5]. The method can recognize the modal parameters of the structure only by the vibration response of the measured structure. However, OMA requires that the excitation force satisfy the "white noise" assumption, but the excitation force generated in the actual working state of the machine is obviously not satisfied with this assumption. We propose an active excitation modal analysis (AEMA) technology to solve this problem. The method can effectively identify the dynamic characteristics of the machine in operation state by designing specific motion law to excite machine tool structure [6].

This paper designs $\mathrm{G}$ code with certain law to control the machine worktable movement. The inertial force generated during the reciprocating motion of the machine table is used to excite the machine tool. So we can obtain the modal parameters of the machine structure in working state. We analyzed the feed speed and acceleration factors that may affect the excitation effect and designed the experiment to verify the effect of the worktable empty running excitation.

\section{Worktable empty running excitation movement design}

Theoretical background. The process of generating and transmitting force of drive system is shown in Fig. 1. CNC system executes the G code, then sends motion command to the servo motor. The 
drive system converts the motor torque into a driving force to drive the worktable to move. When worktable reciprocates, the inertial force generated by worktable acceleration or deceleration is conveyed to all parts of the machine through the screw nut pair - ball screw - bearing - machine body and causes the vibration of the whole machine tool structure[7]. The vibration of the screw drive system can be viewed as vibration of a beam with a moving mass, including axial and torsional vibration. The axial contact deformation between the screw and the nut is the main cause of the screw axial vibration and torsional vibration[8].

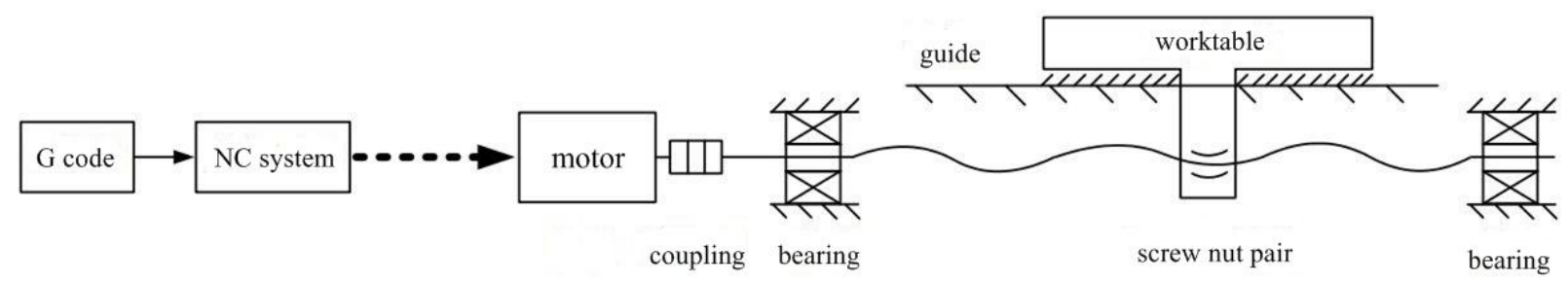

Fig.1 Schematic diagram of the excitation force generating and transmitting

The movement of the CNC machine is controllable. So we can design the worktable movement law to excite the machine structure effectively. CNC machine structure vibration response signal can be picked up through data acquisition system, sensors and other ancillary equipment. Using the responsebased modal analysis algorithm to analyze the vibration response signal, we can identify the modal parameters of the machine. Compared to the traditional method of EMA based on frequency response function (FRF), the method can identify the modal parameters only by using the vibration response signal of the structure in working state[9]. In order to make the excitation meet "white noise" requirement, the inertial force generated by worktable must be random. We control the movement of worktable by designing $\mathrm{G}$ code to make time interval for each excitation force random. So this method can meet the "white noise" excitation in a certain frequency band.

Excitation energy and bandwidth design. As for empty running excitation, the excitation force must have enough energy and bandwidth so as to effectively excite various modes that we need. Every inertial impact caused by worktable acceleration and deceleration can be approximated as a pulse signal. So the random acceleration and deceleration sequence can be seen as a number of random variable pulse signal in time domain. During the reciprocating motion of worktable, it is assumed that the duration time of the single impact is the same. That is the duration time $\tau$ of each pulse remains the same. The single pulse excitation amplitude is $A_{i}$. So the mathematical model of the inertial excitation force sequence can be expressed as follows:

$$
\begin{aligned}
& f_{i}(t)=\left\{\begin{array}{ll}
A_{i} & \left(\mathrm{t} \in\left[t_{i}, t_{i}+\tau\right]\right) \\
0 & \left(\mathrm{t} \notin\left[t_{i}, t_{i}+\tau\right]\right)
\end{array} .\right. \\
& f(t)=\sum_{i=1}^{n} f_{i}(t) .
\end{aligned}
$$

Finite Fourier transform (FFT) of inertial excitation sequence can be expressed as follows:

$$
F_{T}(\omega)=\int_{-T / 2}^{T / 2} f(t) e^{-j \omega t} d t
$$

The auto-power spectrum of the excitation sequence can be expressed as follows:

$$
S_{f f}(\omega, T)=\frac{1}{T} F_{T}^{*}(\omega) F_{T}(\omega)=\frac{1}{T}\left|F_{T}(\omega)\right|^{2} .
$$


Combined with (1)(2)(3)(4), the auto-power spectrum can be written as:

$$
S_{f f}(\omega, T)=\frac{1}{T} \sum_{i=1}^{n}\left|\int_{-T / 2}^{T / 2} A_{i} e^{-j \omega t} d t\right|^{2} .
$$

From Eq.5 we can see that the bandwidth of the excitation sequence is equal to that of the single excitation force. The excitation energy is not only proportional to the density of the inertial excitation sequence but also to the square of the amplitude of the single pulse. So we can control the pulse density, single pulse amplitude and bandwidth to control the incentive effect of the worktable empty running excitation method.

The amplitude of a single pulse is related to the weight of the worktable, the impact speed and the acceleration. The bandwidth of the single pulse is related to the structure and material of the worktable feed system, especially the material of the screw and nut. The material hardness and the duration of the collision determine the bandwidth. The structure and material of a machine are constant, so the bandwidth of the excitation sequence is constant. So we mainly study the effect of impact speed and acceleration on the excitation.

\section{Experimental analysis of influencing factors of excitation effect}

Experimental setup. In order to analyze the impact speed and acceleration which may influence the effect of the excitation, we do the experiment on CNC machine tool. All tests in this paper were conducted on the same machine tool: a horizontal machining center, model number MAG XS211. Vibration response signal was measured by PCB 356A15 three-direction acceleration sensor. All acceleration sensors were connected to an acquisition system (LMS SCADAS Mobile SCM05), and the sampling rate of the system was $1024 \mathrm{~Hz}$. The acceleration sensors are located on worktable, the nut and the supporting bearings as shown in Fig.2.
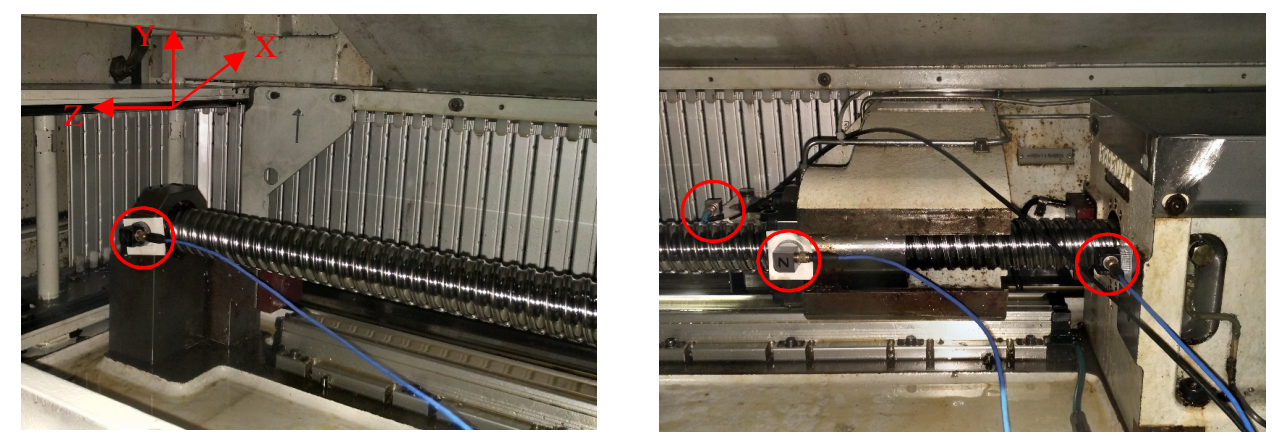

Fig.2 Location of acceleration sensors

The effect of feed speed. In the experiment, the acceleration is constant, the random reciprocating motion of the worktable at different feed speed was designed. We set the acceleration time constant $16 \mathrm{~ms}$ (Time required to accelerate to $1000 \mathrm{~mm} / \mathrm{min}$ ). And set the worktable feed speed $1000 \mathrm{~mm} / \mathrm{min}$, $3000 \mathrm{~mm} / \mathrm{min}, 5000 \mathrm{~mm} / \mathrm{min}, 7000 \mathrm{~mm} / \mathrm{min}, 9000 \mathrm{~mm} / \mathrm{min}$. Fig. 3 shows the sequence of worktable speed and excitation force. The time interval $t i$ between excitation forces is random and generated by MATLAB. The worktable acceleration response signals at different speed are shown in Fig.4.

It can be found that the amplitude of the response signal at different feed speed is different. As the feed speed increases, the excitation energy to the machine structure increases, and the structure response signal amplitude also increases. The structure of machine tool can't be effectively excited if the feed speed is too low. However, it will bring danger to the experiment process if the speed is too high. So on the basis of full excitation and, we should select reasonable worktable feed rate combined with the actual machine tool parameters. 


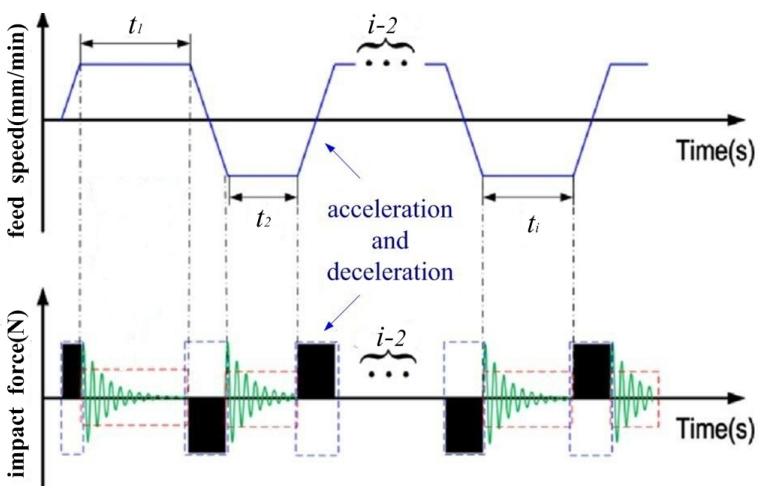

Fig.3 The sequence of worktable speed and excitation forces

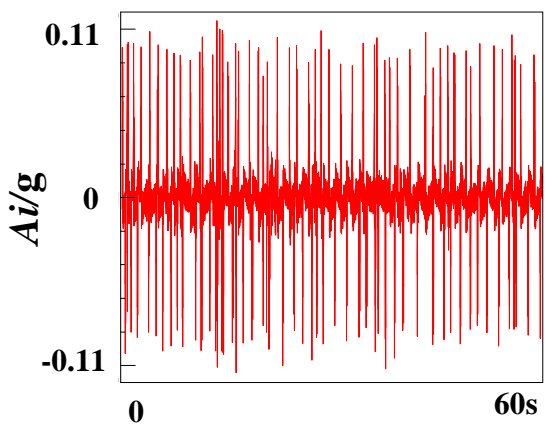

(a) $\mathrm{v}=1000 \mathrm{~mm} / \mathrm{min}$

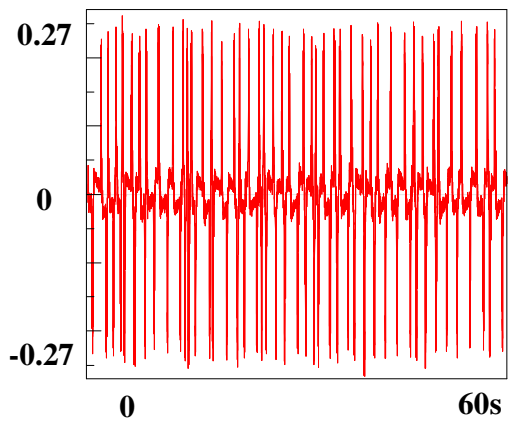

(b) $\mathrm{v}=3000 \mathrm{~mm} / \mathrm{min}$

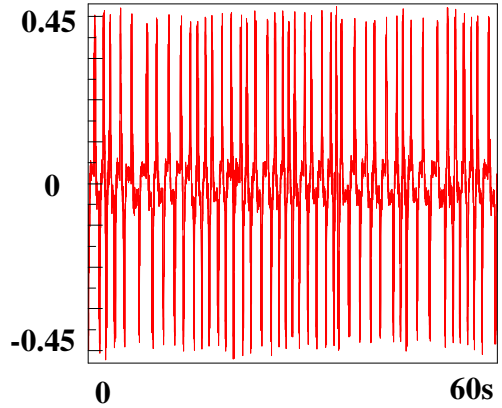

(c) $\mathrm{v}=5000 \mathrm{~mm} / \mathrm{min}$

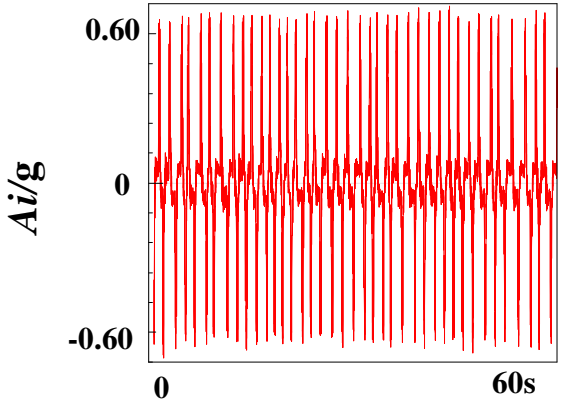

(d) $\mathrm{v}=7000 \mathrm{~mm} / \mathrm{min}$

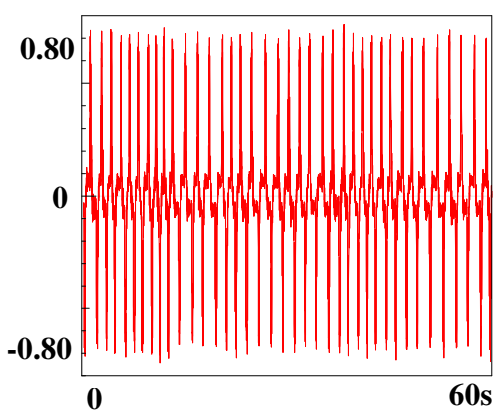

(e) $\mathrm{v}=9000 \mathrm{~mm} / \mathrm{min}$

Fig.4 Acceleration response signals of worktable at different speed

The effect of acceleration. When the worktable weight is constant, the greater the acceleration is, the greater the inertial force is. In this experiment, we kept the feed speed $5000 \mathrm{~mm} / \mathrm{min}$ and set the acceleration time constant $\left(t_{a}\right) 64 \mathrm{~ms}, 32 \mathrm{~ms}, 16 \mathrm{~ms}$. The worktable acceleration response signals at different acceleration are shown in Fig.5.

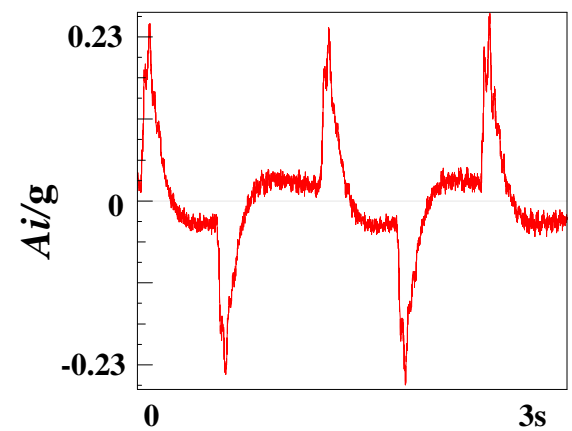

(a) $t_{a}=64 \mathrm{~ms}$

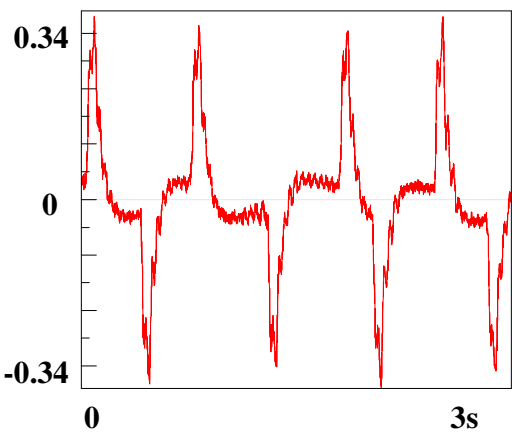

(b) $t_{a}=32 \mathrm{~ms}$

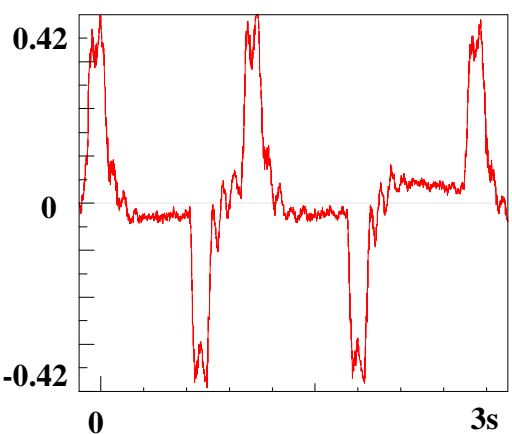

(c) $t_{a}=16 \mathrm{~ms}$

Fig.5 Acceleration response signals of worktable at different acceleration 
It can be found that as the feed speed increases, the structure response signal amplitude also increases. Compared with the feed speed, the change of the response amplitude caused by acceleration is relatively small. Therefore, we mainly control the feed speed to control the excitation energy in the experiment.

\section{Experimental verification}

In order to verify that this method can effectively obtain the dynamic characteristics of the machine structure, we did the worktable empty running experiment. The experiment settings are the same as before. XZ plane worktable of MAG XS211 horizontal machining center reciprocates randomly in Z direction to excite the machine structure. The modal parameters can be identified by the op.PolyMAX algorithm in LMS modal analysis modular. Table 1 shows the natural frequencies of MAG XS211 worktable between $0 \mathrm{~Hz}$ and $900 \mathrm{~Hz}$.

Table 1 Natural frequencies of MAG XS211 worktable

\begin{tabular}{cccccc}
\hline $\begin{array}{c}\text { Speed } \\
(\mathrm{mm} / \mathrm{min})\end{array}$ & $\begin{array}{c}\text { Acceleration time } \\
\text { constant }(\mathrm{ms})\end{array}$ & $\begin{array}{c}\text { Mode1 } \\
(\mathrm{Hz})\end{array}$ & $\begin{array}{c}\text { Mode2 } \\
(\mathrm{Hz})\end{array}$ & $\begin{array}{c}\text { Mode3 } \\
(\mathrm{Hz})\end{array}$ & $\begin{array}{c}\text { Mode4 } \\
(\mathrm{Hz})\end{array}$ \\
\hline \multirow{3}{*}{1000} & 16 & 218.258 & 416.300 & 444.243 & 798.887 \\
& 32 & 218.301 & 416.525 & 441.820 & 800.819 \\
& 64 & 215.713 & 412.206 & 587.399 & 801.980 \\
3000 & 16 & 222.064 & 390.250 & 446.448 & 800.520 \\
& 32 & 219.845 & 387.850 & 446.127 & 796.859 \\
& 64 & 219.869 & 388.537 & 445.533 & 797.683 \\
5000 & 16 & 217.245 & 397.084 & 443.456 & 778.752 \\
& 32 & 217.481 & 394.043 & 446.962 & 783.163 \\
7000 & 64 & 216.790 & 388.615 & 445.136 & 793.295 \\
& 16 & 230.176 & & 443.369 & 785.062 \\
& 32 & 216.444 & 398.197 & 445.013 & 788.435 \\
& 64 & 214.671 & 389.597 & 446.589 & 790.557 \\
9000 & 16 & 228.185 & & 441.594 & 787.863 \\
& 32 & 212.353 & & 444.160 & 784.145 \\
\hline
\end{tabular}

From the natural frequencies summarized in Table 1, it can be seen that three or four modes are excited at any feed speed and acceleration. So this method can effectively excite the machine and identify the modal parameters of the machine structure. The excitation bandwidth is the same at any speed and acceleration. So excitation bandwidth has nothing to do with speed and acceleration. Mode number and mode value are different at different feed speed and acceleration. Therefore it can be concluded that excitation effect is related to speed and acceleration. We should select the appropriate feed speed and acceleration in experiment.

\section{Conclusion}

In this paper, an approach of exciting CNC machine tools based on worktable empty running excitation was studied. The motion of worktable empty running excitation was designed and the influencing factor of excitation was analyzed. Finally, the empty running experiment at different worktable feed speed and acceleration was carried out to verify this approach. It is concluded that: 
1. This method can effectively excite the machine and identify the modal parameters of the machine structure in operation state. Excitation effect is related to worktable feed speed and acceleration.

2. Excitation energy is related to worktable feed speed and acceleration, feed speed has a more important effect than acceleration. Excitation bandwidth is related to the structure and material of the worktable feed system. It has nothing to do with worktable speed and acceleration.

\section{Acknowledgements}

The research is supported by the National Natural Science Foundation of China under Grant No. 51375193 and 51775212, and the National Science and Technology Major Project no. $2014 Z X 04002011$.

\section{References}

[1] Kivanc E B, Budak E. Structural modeling of end mills for form error and stability analysis[J]. International Journal of Machine Tools and Manufacture, 2004, 44(11): 1151 1161.

[2] Altintas Y. Analytical prediction of three dimensional chatter stability in milling[J]. JSME International Journal Series C, 2001, 44(3): 717 723.

[3] Da Silva M M, Brüls O, Swevers J, et al. Computer-aided integrated design for machines with varying dynamics[J]. Mechanism and Machine Theory, 2009, 44(9): 1733-1745.

[4] Brecher C, Esser M, Witt S. Interaction of manufacturing process and machine tool[J]. CIRP Annals-Manufacturing Technology, 2009, 58(2): 588-607.

[5] Zaghbani I, Songmene V. Estimation of machine-tool dynamic parameters during machining operation through operational modal analysis[J]. International Journal of Machine Tools and Manufacture, 2009, 49(12): 947 957.

[6] Li B, Wei Y Q, Mao X Y*, Mao K M, Liu H Q, Tian H L. A novel vibration exciting method for NC machine tools[C]. 2010 International Conference on System Science, Engineering Design and Manufacturing Informattization.

[7] Liu Xiangqiu. Analysis of Dynamic Characteristics of CNC Machine Tool Structure during Working Space Based on Multi-Axis Excitation Technology[M].Wuhan: Huazhong University of Science and Technology,2015.

[8] Paolo Gallina. Vibration in screw jack mechanisms:experimental results[J].Journal of Sound and Vibration,2005,282:1025-1041.

[9] Li, B., et al., A new approach to identifying the dynamic behavior of CNC machine tools with respect to different worktable feed speeds. International Journal of Machine Tools and Manufacture, 2013. 72: 73-84. 